\title{
CD164 regulates proliferation and apoptosis by targeting PTEN in human glioma
}

\author{
MING TU ${ }^{1,2}$, LIN CAI $^{2}$, WEIMING ZHENG ${ }^{2}$, ZHIPENG SU $^{2}$, YONG CHEN $^{3}$ and SONGTAO QI ${ }^{1}$ \\ ${ }^{1}$ Department of Neurosurgery, NanFang Hospital of Southern Medical University, Guangzhou, Guangdong 510515; \\ ${ }^{2}$ Department of Neurosurgery, The First Affiliated Hospital of Wenzhou Medical University, Wenzhou, Zhejiang 325000; \\ ${ }^{3}$ Department of Neurosurgery, The Second People's Hospital of Yueyang, Yueyang, Hunan 414000, P.R. China
}

Received November 30, 2015; Accepted December 22, 2016

DOI: $10.3892 / \mathrm{mmr} .2017 .6204$

\begin{abstract}
Cluster of differentiation 164 (CD164), a sialomucin, has been demonstrated to be involved in the regulation of proliferation, apoptosis, adhesion and differentiation in multiple cancers. CD164 is regarded to be a potential promotor of tumor growth. However, the involvement of CD164 in human glioma proliferation and apoptosis remains unknown. The aim of the present study was to investigate the expression and oncogenic function of CD164 in normal human astrocytes (NHA) and glioma cells in vitro and in vivo. The results of the present study demonstrated that CD164 mRNA and protein levels were significantly increased in human glioma cell lines and tissue samples. CD164 overexpression promoted the proliferation of NHA in vitro, and its tumorigenic effect was confirmed in a murine xenograft model. Knockdown of CD164 inhibited cell proliferation and promoted apoptosis of the U87 human glioma cell line in vitro and in vivo. In addition, knockdown of CD164 was demonstrated to upregulate the $\mathrm{Bax} / \mathrm{Bcl} 2$ ratio and phosphatase and tensin homolog (PTEN) expression, reduce protein kinase $\mathrm{B}$ (AKT) phosphorylation and promote the expression of p53 in U87 cells. The results suggest that CD164 expression may have affected the proliferation and apoptosis of human glioma cells via the PTEN/phosphoinositide 3-kinase/AKT pathway, and may therefore present a potential target for the diagnosis and treatment of glioma.
\end{abstract}

Correspondence to: Dr Songtao Qi, Department of Neurosurgery, NanFang Hospital of Southern Medical University, 1838 Guangzhou Avenue North Road, Guangzhou, Guangdong 510515, P.R. China E-mail: songtaoqi2015@sina.com

Abbreviations: NHA, normal human astrocytes; shRNA, short hairpin RNA; CCK-8, cell counting kit-8; RT-qPCR, reverse transcription-quantitative polymerase chain reaction; $\mathrm{BC}$, blank control; NC, negative control; TUNEL, terminal deoxyribonucleotidyl transferase-mediated dUTP nick end labeling

Key words: glioma, cluster of differentiation 164, proliferation, apoptosis, phosphatase and tensin homolog

\section{Introduction}

Gliomas are the most common primary brain tumors that arise from the neuroectoderm (1). Despite significant advances in complete surgery, radiotherapy and chemotherapy, the survival time of patients remains $\sim 12$ months following diagnosis (2). Therefore, the identification of a potential biological target that increases the likelihood of patients with glioma achieving remission remains a priority. Furthermore, the majority of prognostic factors provide no insight into the molecular events that are responsible for tumor proliferation, apoptosis or additional biological properties of malignancy $(3,4)$. Currently, emerging novel targeted therapies, such as genetic treatment and immunological therapy, may provide alternative strategies for the treatment of glioma (5).

The cluster of differentiation 164 (CD164) glycoprotein is a member of the sialomucin family, which is a mucin that contains sialic acid (6). CD164 was first identified as a carrier of a peanut agglutinin-binding site, which is a tumor-associated carbohydrate marker expressed in human gastric carcinoma cells and bone marrow stromal reticular cells (7-9). Previous studies have demonstrated that CD164 modulates the proliferation, adhesion and migration of hematopoietic stem and progenitor cells $(10,11)$. It has been reported that CD164 regulates hematopoiesis by facilitating the adhesion of human $\mathrm{CD}_{3} 4^{+}$cells to bone marrow stroma (12). In addition, CD164 has been demonstrated to regulate the growth and differentiation of normal cells, and is involved in malignant cell proliferation as well as invasion (13). Furthermore, CD164 has been implicated in the maintenance and progression of multiple human solid cancers, including medulloblastoma, ovarian (14) and colon (15) cancers. A previous study demonstrated that CD164 may participate in the mediation of prostate cancer bone metastasis (16). In addition, CD164 has been recognized as a biomarker for the detection of acute lymphoblastic leukemia and allergy $(17,18)$. These studies indicated that CD164 may function as a key molecule in the modulation of tumor progression. However, the role of CD164 in human glioma has yet to be elucidated. The present study investigated the expression profile of CD164 in glioma cells, and examined the correlation between CD164 and tumorigenesis of glioma cells in vitro and in vivo, including the proliferation and apoptosis levels. 


\section{Materials and methods}

Patients and tissue specimens. The ethics committee of The First Affiliated Hospital of Wenzhou Medical University (Wenzhou, China) approved the protocols employed in the present study (October, 2012). Samples consisted of 50 paired glioma and adjacent normal brain tissue samples (24 males and 26 females) admitted to the Department of Neurosurgery of The First Affiliated Hospital of Wenzhou Medical University (Wenzhou, China) between December 2013 and December 2015. The age of the patients ranged from 46 to 73 with a mean of $63 \pm 5$ years. All cases were histologically confirmed by trained pathologists. No patients had received chemotherapy or radiotherapy prior to surgery, and informed consent was obtained from all patients.

Cell culture. HEK-293T cells, three human glioma cell lines (U251, SHG-44 and U87) and normal human astrocytes (NHA) were purchased from the American Type Culture Collection (Manassas, VA, USA). All cell lines were cultured in Dulbecco's modified Eagle's medium (Gibco; Thermo Fisher Scientific, Inc., Waltham, MA, USA) containing 10\% fetal bovine serum (Gibco; Thermo Fisher Scientific, Inc.) and maintained at $37^{\circ} \mathrm{C}$ and $5 \% \mathrm{CO}_{2}$.

Lentiviral infection. Short hairpin RNA (shRNA) specifically targeting human CD164 and negative scrambled control shRNA were purchased from Thermo Fisher Scientific, Inc. (Waltham, MA, USA). There were 19 nucleotides of the target sequence in the shRNA expression cassette prior to the loop sequence (TTCAAGAGA). The shRNA sequences were as follows: CD164, sense, 5'-TGAGAAAGCTCTCCACTCTGTTCAAGA GACAGAGTGGAGAGCTTTCTCTTTTTTC-3', and antisense, 5'-ACTCTTTCGAGAGGTGAGACAAGTTCTCTG TCTCACCTCTCGAAAGAGAAAAAAGAGCT-3'; negative control, sense, 5'-TAACTAGTAACGGCTGCTCCTTCAA GAGAGGAGCAGCCGTTACTAGTTTTTTTTC-3', and antisense, 5'-ATTGATCATTGCCGACGAGGAAGTTCTCTCC TCGTCGGCAATGATCAAAAAAAAGAGCT-3'. Lentiviruses were produced by cotransfection of the lentiviral packaging plasmids pMD.G and pMDLgpRRE (Ambion; Thermo Fisher Scientific, Inc.) into HEK-293T cells using calcium phosphate. A total of $5 \times 10^{5} \mathrm{U} 87$ cells were transfected with $20 \mu \mathrm{g}$ recombinant lentivirus-transducing units plus $6 \mathrm{mg} / \mathrm{ml}$ polybrene (BD Biosciences, San Jose, CA, USA). A total of $5 \times 10^{5}$ U87 cells overexpressing CD164 were established by transfection with the lentivirus-expressing pRSVRev-vector with the human CD164 coding sequence (Ambion; Thermo Fisher Scientific, Inc.) at a concentration of $5 \times 10^{9} \mathrm{Tu} / \mathrm{ml}$. The blank control cells were treated with PBS.

Cell proliferation. Cells were diluted to a density of $2 \times 10^{4}$ cells $/ \mathrm{ml}$, and $100 \mu \mathrm{l}$ cell solution was transferred to each well of 96-well culture plates and incubated for 24,48 or $72 \mathrm{~h}$. Cell proliferation was then assessed using the Cell Counting kit-8 assay (CCK-8; Dojindo Molecular Technologies, Inc., Kumamoto, Japan), according to the manufacturer's protocol. Following incubation with $10 \mu \mathrm{l} \mathrm{CCK}-8$ solution at $37^{\circ} \mathrm{C}$ for $60 \mathrm{~min}$ in a $\mathrm{CO}_{2}$ incubator, the absorbance at $490 \mathrm{~nm}$ was measured using a microplate spectrophotometer (BioTek
Instruments, Inc., Winooski, VT, USA). This experiment was repeated twice.

Annexin V/propidium iodide (PI) staining assay. To determine the extent of early apoptosis and late apoptosis/necrosis in cells, an Annexin V-FITC/PI apoptosis detection kit (BD Biosciences) was used according to the manufacturer's protocol. A total of $\geq 10,000$ cells were analyzed for each sample. The proportion of U87 cells in early apoptosis and late apoptosis/necrosis were calculated by recording the percentage of Annexin $\mathrm{V}^{+} / \mathrm{PI}^{-}$and Annexin $\mathrm{V}^{+} / \mathrm{PI}^{+}$-labeled cells, respectively. The stained cells were analyzed directly by flow cytometry using the FACS Calibur machine (BD Biosciences) using the Cell Quest program (BD Biosciences) for data analysis.

Reverse transcription-quantitative polymerase chain reaction $(R T-q P C R)$ analysis. Total RNA was extracted from the tissue samples and $5 \times 10^{6}$ U87 cells using TRIzol reagent (Thermo Fisher Scientific, Inc.) according to the manufacturer's protocol. RNA concentrations were determined by spectrophotometry (DU-800; Beckman Coulter). A 260/280 absorbance ratio of 1.96 implied clean RNA at a concentration of $0.23 \mathrm{mg} / \mathrm{ml}$. Total $1 \mu \mathrm{g}$ RNA was reverse transcribed to cDNA using the PrimeScript RT Reagent kit (Applied Biosystems; Thermo Fisher Scientific, Inc.), according to the manufacturer's instructions. qPCR was performed using the ABI 7300 Real-Time PCR system (Applied Biosystems; Thermo Fisher Scientific, Inc.) with the following amplification conditions: $30 \mathrm{sec}$ at $98^{\circ} \mathrm{C}, 30$ cycles of $10 \mathrm{sec}$ at $98^{\circ} \mathrm{C}, 15 \mathrm{sec}$ at $60^{\circ} \mathrm{C}, 15 \mathrm{sec}$ at $72^{\circ} \mathrm{C}$ and $2 \mathrm{~min}$ at $72^{\circ} \mathrm{C}$. The primer sequences were as follows: CD164, forward, 5'-TGAGCCCTGAACACCAGAGAG-3', and reverse, 5'-AAAGCCAGATGAGCGCTTCTA-3'; phosphatase and tensin homolog (PTEN), forward, 5'-TCGTGGGTGCCT CGCT-3', and reverse, 5'-CACCACTACAGCCAGCATTTT C-3'; GAPDH, forward, 5'-AACGGATTTGGTCGTATT GGG-3', and reverse, 5'-TCGCTCCTGGAAGATGGTGAT-3'. The expression target genes in all samples was normalized to GAPDH. Following data collection, target gene expression was quantified by relative quantitative analysis using the $2^{-\Delta \Delta C \mathrm{C}}$ method as described previously (19).

Western blot analysis. A total of $5 \times 10^{6} \mathrm{U} 87$ or NHA cells were washed and lysed with lysis buffer $(20 \mathrm{mmol} / 1$ Tris- $\mathrm{HCl}$ (pH 7.4), $100 \mathrm{mmol} / \mathrm{l} \mathrm{NaCl}, 1 \% \mathrm{NP} 40,0.5 \%$ sodium deoxycholate, $5 \mathrm{mmol} / 1 \mathrm{MgCl}_{2}, 0.1 \mathrm{mmol} / \mathrm{l}$ phenylmethylsulfonyl fluoride, and $10 \mathrm{mg} / \mathrm{ml}$ protease inhibitor mixture) from Nanjing KeyGen Biotech. Co., Ltd. (Nanjing, China). The suspension was centrifuged at $5,000 \times \mathrm{g}$ at $4^{\circ} \mathrm{C}$ for $10 \mathrm{~min}$, followed by centrifugation at $16,000 \times \mathrm{g}$ at $4^{\circ} \mathrm{C}$ for $30 \mathrm{~min}$, and then the supernatant was collected and kept at $-70^{\circ} \mathrm{C}$ until use. Whole cell proteins were extracted using Mammalian Protein Extraction Reagent (Pierce; Thermo Fisher Scientific, Inc.), while protein concentrations were measured using a bicinchoninic acid assay kit (Pierce; Thermo Fisher Scientific, Inc.). Equal amounts of total protein (20-40 $\mu \mathrm{g})$ were electrophoresed in an 8\% SDS-PAGE gel with Tris-glycine, before they were transferred to a nitrocellulose membrane. The membranes were then blocked with Tris-buffered saline containing 5\% non-fat milk powder at room temperature for 
$1 \mathrm{~h}$, and were incubated with specific antibodies for CD164 (catalog no. C9618; 1:500), Bax (catalog no. B8429; 1:1,000), Bcl2 (catalog no SAB4300339; 1:1,000), caspase3 (catalog no. SAB4503292; 1:1,000), PTEN (catalog no. SAB4300337; 1:1,000), p53 (catalog no. P9249; 1:1,000), total AKT (catalog no. SAB4500799; 1:1,000) and phospho-AKT (catalog no. SAB4503853; 1:1,000), all from Sigma-Aldrich, Merck KGaA, Darmstadt, Germany. The membranes were subsequently probed with anti-rabbit $\lg$ (catalog no. A0545; 1:5,000) or anti-mouse lgG (catalog no. SAB3701044; 1:5,000) secondary antibody conjugated with horseradish peroxidase (Sigma-Aldrich, Merck KGaA) at room temperature for $1 \mathrm{~h}$. Band signals were detected using an enhanced chemiluminescence kit (Pierce; Thermo Fisher Scientific, Inc.) and immunoreactive bands were quantified using Alphaimager 2200 (Alpha Innotech, San Leandro, CA, USA). $\beta$-actin was used as the internal control.

In vivo tumorigenesis. In vivo experiments were conducted as described previously (20). A total of 50 male athymic BALB/c nu/nu mice (age, 4-6 weeks) were obtained from the Shanghai Experimental Center, Chinese Science Academy (Shanghai, China) and maintained under pathogen free conditions in a temperature and humidity controlled animal care facility with a $12 \mathrm{~h}$ light dark cycle. Mice were allowed access to sterile food and water ad libitum. NHA infected with vector control or CD164 lentivirus were injected subcutaneously into the flank of nude mice at a dose of $1 \times 10^{7}$ cells/mouse. A $100 \mu \mathrm{l}$ aliquot of the U87 cell suspension (equivalent to $1 \times 10^{7}$ U87 cells) was injected into the flank of nude mice in the corresponding group. Following 56 days of tumor growth, the experiment was terminated and mice with subcutaneous tumors were sacrificed by cervical dislocation.

Immunolocalization of the Ki-67 marker of proliferation in tumor samples. Paraffin-embedded subcutaneous xenograft tissue sections were fixed with $4 \%$ paraformaldehyde at room temperature for $24 \mathrm{~h}$. Following washing in phosphate-buffered saline, the endogenous peroxidase activity of slides was blocked with protein blocking solution (Dako, Glostrup, Denmark) at room temperature for $30 \mathrm{~min}$. For Ki-67 immunohistochemistry, the samples were first incubated with a primary antibody against Ki-67 (catalog no. SAB5500134; 1:100) overnight at $4^{\circ} \mathrm{C}$ (Sigma-Aldrich, Merck KGaA), followed by incubation with an appropriate anti-rabbit $\lg \mathrm{G}$ (catalog no. A0545; 1:5,000; Sigma-Aldrich, Merck KGaA) at room temperature for $2 \mathrm{~h}$. The immunogenicity of slides was detected using the Vecstain ${ }^{\mathrm{TM}} \mathrm{ABC}$ kit (Vector Laboratories, Burlingame, California, USA) according to the manufacturer's protocol. The stained slides were analyzed under a light microscope (Olympus Corporation, Tokyo, Japan), and were analyzed using the Image-Pro Plus software system version 6.0 (Media Cybernetics, Inc., Rockville, MD, USA). A total of 20 fields of view were assessed by an investigator who was blinded to the experimental data.

Terminal deoxyribonucleotidyl transferase-mediated dUTP nick end labeling (TUNEL) assay. The number of apoptotic cells in the subcutaneous xenograft tumors was studied using an in situ cell death detection kit purchased from Roche
Diagnostics (Basel, Switzerland), which was performed according to the manufacturer's protocol. Counterstaining was performed with hematoxylin (Nanjing Keygen Biotech Co., Ltd.) at room temperature for $1 \mathrm{~min}$. The tissue sections were mounted under a glass coverslip and viewed under a light microscope by two different pathologists unaware of the xenograft tumor groups. The apoptotic cells were counted in 20 randomly selected fields of the most affected tumor areas under x400 magnification.

Statistical analysis. All data were expressed as the mean \pm standard deviation for the absolute values or percentages of controls. Data were evaluated by one-way analysis of variance followed by Student-Newman-Keuls-q multiple comparisons tests using SPSS software (version 17.0; SPSS, Inc. Chicago, IL, USA, ). $\mathrm{P}<0.05$ was considered to indicate a statistically significant difference.

\section{Results}

Analysis of CD164 in glioma cell lines and clinical specimens. CD164 mRNA expression levels in U251, SHG-44 and U87 glioma cell lines was significantly higher when compared with NHA cells $(\mathrm{P}<0.001, \mathrm{P}<0.001$ and $\mathrm{P}<0.001$, respectively; Fig. 1A). In addition, CD164 protein expression levels were determined in U251, SHG-44 and U87 glioma cell lines, and the NHA cell line by western blot analysis. CD164 expression was almost undetectable in NHA cells and was visibly elevated in U251, SHG-44 and U87 cells by comparison (Fig. 1B). As the level of CD164 expression in U87 cells was significantly upregulated in contrast to U251 and SHG-44 cells, U87 cells were selected for subsequent experiments. To further validate the expression of CD164 in glioma in vivo, the expression of CD164 in 50 gliomas and normal adjacent tissue samples was analyzed by RT-qPCR analysis. Compared with normal brain tissues, the glioma tissues demonstrated a significant increase in CD164 mRNA expression levels ( $\mathrm{P}<0.001$; Fig. 2), indicating that CD164 may be associated with the development and progression of glioma.

Overexpression of CD164 promotes cell growth of NHA cells in vitro and in vivo. In order to determine the transforming effect of CD164 in normal cells, CD164 was overexpressed in NHA cells (Fig. 3A). Ectopic expression of CD164 significantly promoted cell growth in NHA cells when compared with the vector control cells, as determined using the CCK-8 assay (Fig. 3B). A previous study demonstrated that the $\mathrm{Bcl}-2$-associated $\mathrm{X}$, apoptosis regulator $(\mathrm{Bax}) / \mathrm{B}$ cell lymphoma $2(\mathrm{Bcl} 2)$ ratio represents the critical balance of regulatory pro-apoptotic and anti-apoptotic proteins in the process of apoptosis (21). As expected, visible increases in $\mathrm{Bcl} 2$ protein levels and decreases in Bax and PTEN protein levels were detected in NHA cells overexpressing CD164 (Fig. 3A). These results suggest that CD164 may modulate NHA progression by increasing cell proliferation, which is considered to be associated with a significant decrease in the $\mathrm{Bax} / \mathrm{Bcl} 2$ ratio (22). Cell viability was significantly increased in NHA cells overexpressing CD164 when compared with the vector control at 24, 48 and $72 \mathrm{~h}$ following transfection $(\mathrm{P}<0.01, \mathrm{P}<0.001$ and $\mathrm{P}<0.001$, respectively; Fig. $3 \mathrm{~B})$. Based on 
A

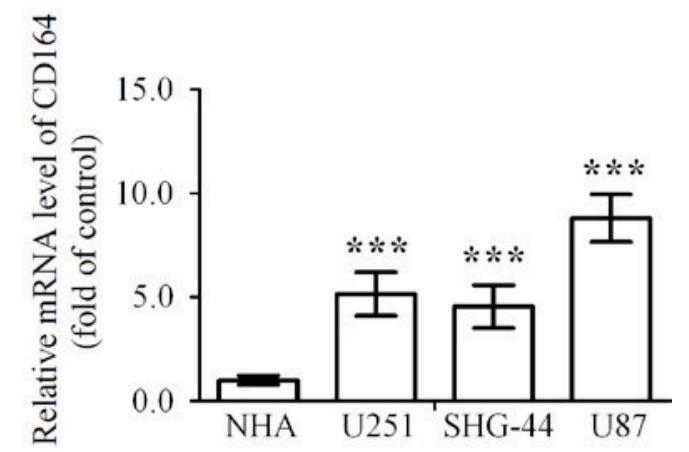

B

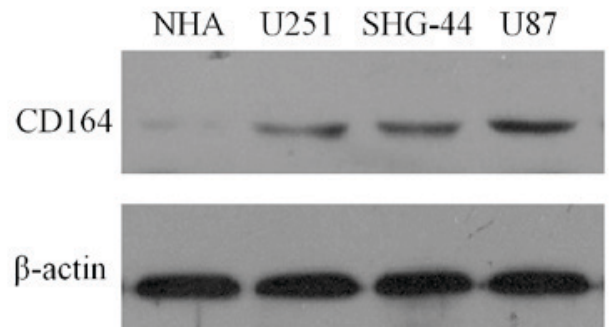

Figure 1. CD164 expression was increased in glioma U251, SHG-44 and U87 cells when compared with NHA cells, as determined using (A) RT-qPCR and (B) western blot analyses. GAPDH was used as a control for RT-qPCR analysis and $\beta$-actin was used as a loading control for western blotting. ${ }^{* * * *} \mathrm{P}<0.001$ vs. NHA. CD164, cluster of differentiation 164; NHA, normal human astrocytes; RT-qPCR, reverse transcription-quantitative polymerase chain reaction.

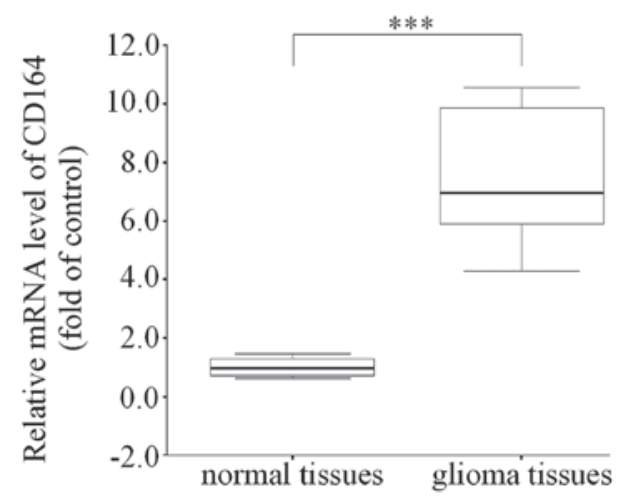

Figure 2.CD164 mRNA expression levels in glioma tissues were significantly higher when compared with paired adjacent normal brain tissues. ${ }^{* * *} \mathrm{P}<0.001$ vs. normal tissues. CD164, cluster of differentiation 164.

these results, it was hypothesized that CD164 may be involved in promoting tumor growth in vivo. Therefore, a subcutaneous tumor xenograft model in nude mice was generated to assess the tumor formation ability of NHA-CD164 cells, which was compared with U87 cells as a positive control. Subcutaneous injection of NHA-CD164 cells and U87 cells lead to tumor formation in all nude mice. NHA-CD164 and U87 tumors were evident at 10 days following implantation, whereas NHA-vector control tumors were too small to be measured at 56 days following implantation. The average weight of the tumors formed by the subcutaneous injection of NHA-CD164 cells was significantly higher than those formed by U87 cells (1294.5 \pm 385.2 mg vs. 637.2 \pm 113.8 mg; P<0.001; Fig. 3C).
Downregulation of CD164 inhibits the proliferation of glioma cells. In order to investigate the function of CD164 in tumorigenesis in vivo, its expression was silenced in U87 cells by tumor-specific lentivirus-mediated shRNA targeting of the CD164 gene. The U87 cells with silenced CD164 expression were then used to determine whether this was associated with inhibition of cell proliferation in vitro. The mRNA and protein levels of CD164 in U87 cells were analyzed by RT-qPCR and western blot analyses, respectively. Transfection of CD164 shRNA resulted in a significant downregulation in CD164 mRNA expression levels when compared with those transfected with negative control shRNA ( $\mathrm{P}<0.001$; Fig. 4A). In addition, protein expression levels were markedly decreased in shCD164-tranfected cells when compared with negative control shRNA or blank control cells (Fig. 4B). CD164 mRNA and protein expression levels were not significantly altered between cells transfected with negative control shRNA and blank controls (Fig. 4A and B).

The effect of downregulated CD164 expression on the proliferation of U87 cells was then investigated further. U87 cell viability was decreased by $45.8 \pm 7.5 \%$ in response to CD164 knockdown when compared with cells transfected with negative control shRNA or blank control cells ( $\mathrm{P}<0.01$; Fig. 4C). In addition, the involvement of CD164 in the apoptosis of U87 cells was assessed using an Annexin V/PI assay. Silenced CD164 expression was associated with a marked increase in the percentage of apoptotic U87 cells compared with BC or NC group, demonstrating a statistically significant difference (Fig. 4D). It has been previously reported that caspase 3 is involved in the terminal phase of apoptosis (23). Therefore, the activation of apoptosis was investigated in the present study by measuring caspase 3 cleavage in U87-shCD164 cells. Knockdown of CD164 expression was associated with a visible increase in the protein expression levels of cleaved caspase 3 (Fig. 4B). In addition, the results demonstrated that downregulation of CD164 visibly reduced the level of Bcl2 and promoted the expression of Bax (Fig. 4B).

CD164 shRNA inhibits tumor growth in a nude mouse xenograft tumor model. In order to elucidate the potential tumorigenic function of CD164 in glioma cells in vivo, a xenograft tumor model was established to compare the tumorigenesis of U87 cells with or without transfection of CD164 shRNA. As shown in Fig. 5A, the final tumor weights were significantly lower in the U87-shCD164 group when compared with the negative control shRNA group ( $>50 \%$ reduction, $\mathrm{n}=8$; $\mathrm{P}<0.001)$. Furthermore, the average weight of tumors in the negative control shRNA group was not statistically different from the blank control group (Fig. 5A).

Tumor immunohistochemistry in vivo. In order to examine the expression of Ki-67 in the tumor tissues, immunohistochemical staining was performed. Tumors from the U87-shCD164 group demonstrated visibly lower positive immunohistochemical staining for Ki-67 when compared with the negative control shRNA and blank control groups (Fig. 5B). A TUNEL staining assay for apoptosis was subsequently performed in these tumor tissues. The rate of apoptosis was visibly increased in the U87-shCD164 group when compared with the negative control shRNA and blank control group (Fig. 5C), which 
A

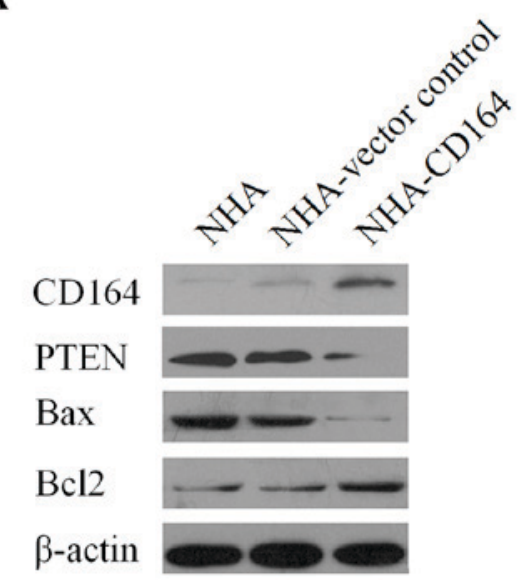

B

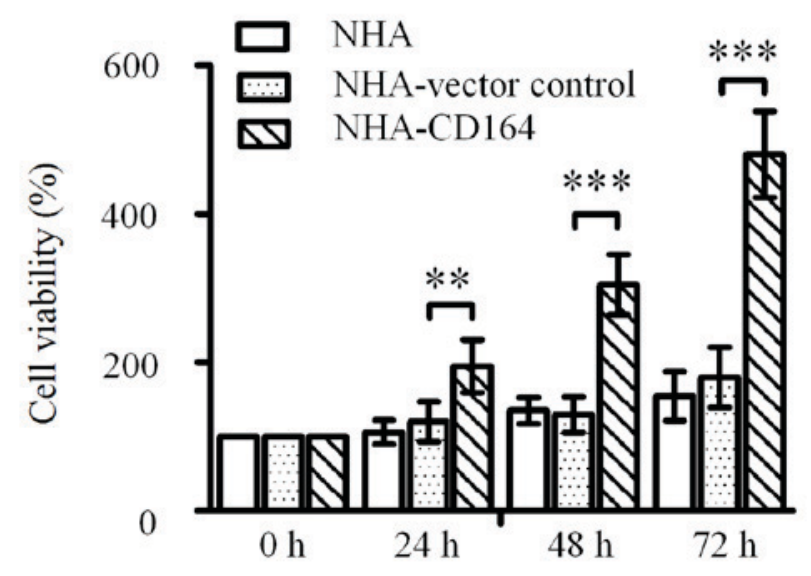

C

NHA-vector control

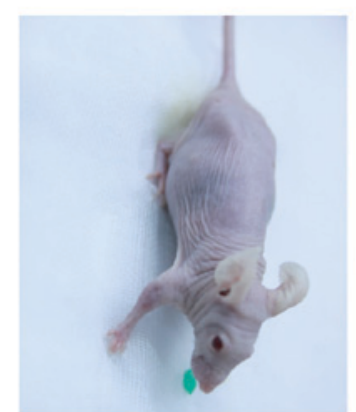

NHA-CD164

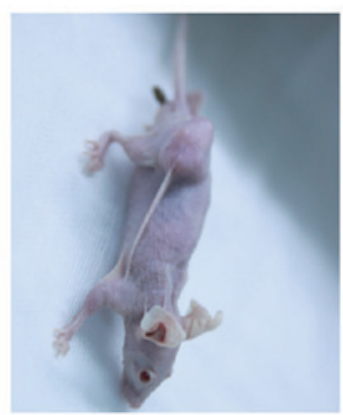

U87

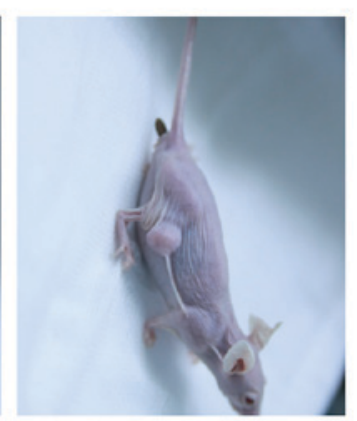

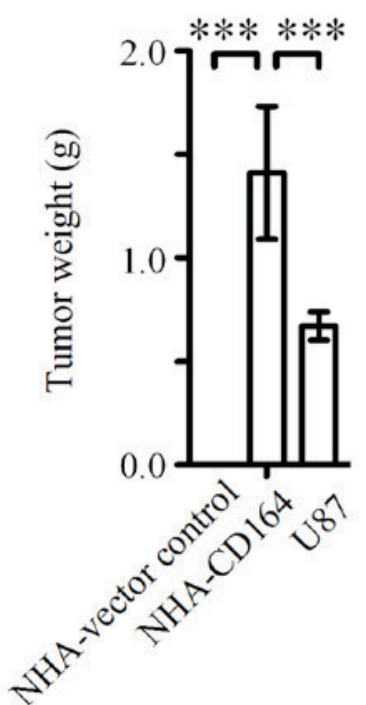

Figure 3. Effect of CD164 on the cell growth of NHA cells in vitro and in vivo. (A) Western blot analysis of CD164, PTEN, apoptotic Bax and anti-apoptotic Bcl2 proteins in NHA-CD164 cell lysates. $\beta$-actin was used as a loading control. ${ }^{* *} \mathrm{P}<0.01$ and ${ }^{* * *} \mathrm{P}<0.001$ vs. NHA-vector control. (B) The effect of $\mathrm{CD} 164$ overexpression on NHA proliferation, as determined using the Cell Counting kit-8 assay. (C) Macroscopic appearance and quantitative analysis of xenograft tumors following subcutaneous injection of NHA-vector control, NHA-CD164 and U87 cells in the flank of nude mice. ${ }^{* * *} \mathrm{P}<0.001$ as indicated. CD164, cluster of differentiation 164; NHA, normal human astrocytes; PTEN, phosphatase and tensin homolog; Bax, Bcl-2-associated X, apoptosis regulator; Bcl2, B cell lymphoma 2 .

suggests that downregulation of CD164 inhibited glioma growth and promoted glioma apoptosis in vivo.

CD164 shRNA upregulated PTEN and inhibited the phosphoinositide 3-kinase (PI3K)/AKT pathway. To further investigate the molecular mechanisms underlying the involvement of CD164 in the regulation of glioma growth and apoptosis, the levels of PTEN and its downstream targets were assessed by western blot analysis in U87 cells transfected with CD164 shRNA. PTEN and p53 protein expression levels were markedly increased in U87 cells following CD164 shRNA transfection compared with cells transfected with negative control shRNA or blank control cells (Fig. 6A). By contrast, the level of AKT phosphorylation was notably decreased in cells following CD164 shRNA transfection when compared with cells transfected with negative control shRNA or blank control cells (Fig. 6A). Further investigation was conducted to determine whether CD164 shRNA transfection increases PTEN
mRNA expression levels in U87 cells. As shown in Fig. 6B, silencing of CD164 in U87 cells significantly increased the expression of PTEN mRNA compared with cells transfected with negative control shRNA $(\mathrm{P}<0.001)$. Western blot analysis was subsequently performed to determine whether CD164 shRNA-mediated suppression of xenograft tumor growth in mice was associated with upregulation of PTEN in tumors, as observed in cultured cells. PTEN protein levels were markedly increased in tumors from the U87-shCD164 mice when compared with those from the control groups (Fig. 6C). In addition, there was a visible increase in p53 protein levels in U87-shCD164 xenograft tumors tissues compared with the controls (Fig. 6C). These alterations in PTEN and p53 protein levels in U87-shCD164 mice were consistent with the observed expression alterations of these proteins in the cultured cells following transfection with CD164 shRNA (Fig. 6A). These results indicate that the tumorigenicity of CD164 in glioma cells may be mediated by the PTEN/PI3K/AKT pathway. 
A

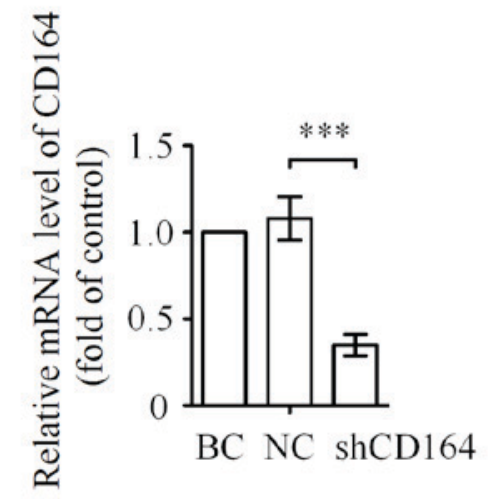

B

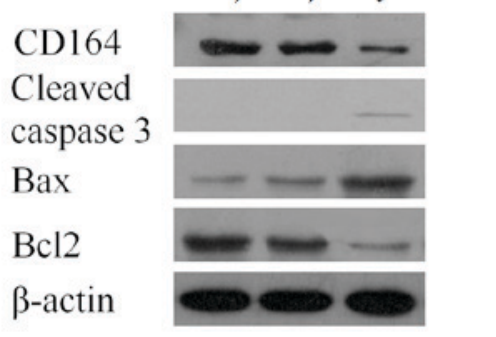

C

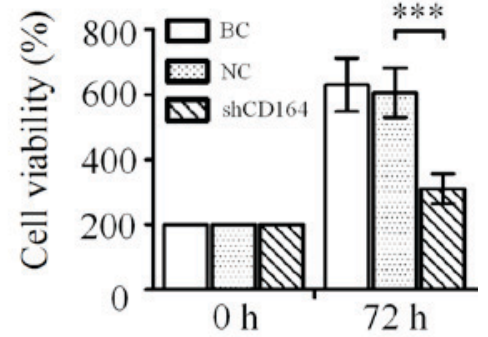

D

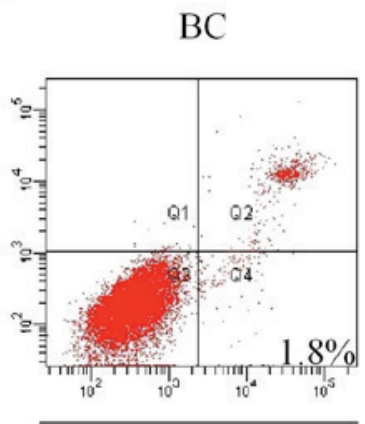

$0 \mathrm{~h}$

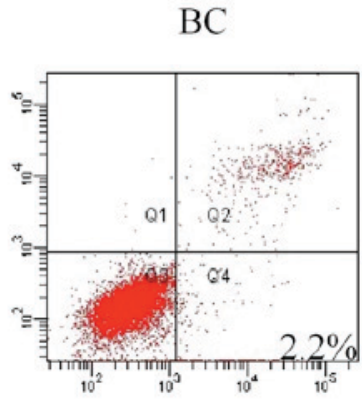

NC

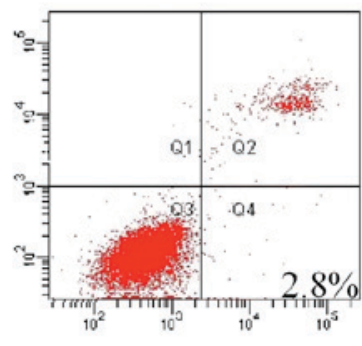

shCD164

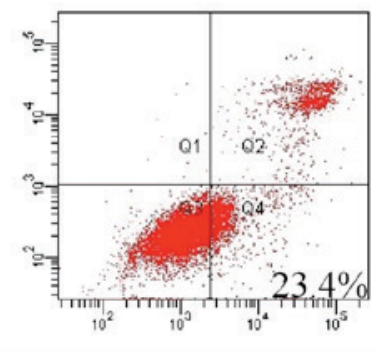

$72 \mathrm{~h}$

Figure 4. Silencing of CD164 inhibits the proliferation of U87 cells in vitro. (A) Knockdown of CD164 mRNA as determined by reverse transcription-quantitative polymerase chain reaction analysis. ${ }^{* * *} \mathrm{P}<0.001$ vs. NC. (B) Western blot analysis of CD164, cleaved caspase 3 , Bax and Bcl2 following transfection with CD164 shRNA. (C) Effect of CD164 shRNA on the proliferation of U87 cells. ${ }^{* *} \mathrm{P}<0.01$ vs. NC. (D) U87 cells were processed for Annexin V/PI staining and analyzed by flow cytometry following transfection with CD164 shRNA for $72 \mathrm{~h}$. CD164, cluster of differentiation 164; Bax, Bcl-2-associated X, apoptosis regulator; Bcl2, B cell lymphoma 2; shRNA, short hairpin RNA; PI, propidium iodide; BC, blank control; NC, negative control.

\section{Discussion}

RNA interference (RNAi) techniques have been proven to be a powerful tool for identifying novel and unexpected tumor promotors and suppressors. Multiple previous studies have used the RNAi approach for the treatment of cancer, in particular those caused by overexpression of oncogenes (14). The present study utilized the RNAi approach to investigate the tumorigenic function of CD164 in glioma. The results of the present study demonstrated that the level of CD164 was increased in malignant glioma cells when compared with normal cells. In addition, RT-qPCR results revealed that CD164 expression was higher in glioma samples when compared with paired normal adjacent brain tissues. This differential expression of CD164 suggests that CD164 may be involved in glioma malignancy. Previous studies have demonstrated that CD164 expression is upregulated in colon cancer, which suggests that CD164 may function as a tumor promoter, and may therefore present a potential target for the treatment of cancer (16). These results are consistent with those presented in the current study.

Previous studies have revealed that CD164 is involved in cancer development via the regulation of cell apoptosis and survival in several cancers (14-18). In addition, CD164 has been implicated in the regulation of human ovarian cancer invasion, and silencing of CD164 expression significantly decreased the metastasis of ovarian cancer cells (20). In the present study, CD164 expression levels were upregulated in NHA cells to investigate the tumorigenic effects of CD164 on NHA cells. The results demonstrated that the overexpression of CD164 promotes the growth of NHA cells in vitro and in vivo, which supports the hypothesis that CD164 may function as a tumor promoter. Previous studies have reported that CD164 is a potential oncogene, based on its ability to target the C-X-C motif chemokine receptor $4(15,20)$. In the present study, CD164 was implicated in the regulation of NHA proliferation and apoptosis by suppressing Bax expression and promoting Bcl-2 expression.

To further elucidate the tumorigenicity of CD164 in glioma, a CD164-silenced cell line was successfully generated by infecting lentivirus CD164 shRNA into U87 cells. The results demonstrated that $\mathrm{CD} 164$ was associated with glioma cell growth. Downregulation of CD164 inhibited cell growth and induced cell apoptosis in U87 cells. Previous studies have indicated that the $\mathrm{Bax} / \mathrm{Bcl} 2$ ratio is involved in the release of cytochrome $\mathrm{c}$ to the cytosol, resulting in caspase activation (21). In addition, overexpression of the anti-apoptotic $\mathrm{Bcl} 2$ protein protects cells from apoptosis induced by stimulants (22). The present study demonstrated that silencing of CD164 in U87 cells results in upregulation of cleaved caspase 3 and alters the $\mathrm{Bax} / \mathrm{Bcl} 2$ ratio by increasing $\mathrm{Bax}$ and decreasing $\mathrm{Bcl} 2$ expression. These in vitro results indicated that a decrease in CD164 expression may have participated in the apoptosis of U87 cells.

To further investigate whether the anti-proliferative effects of CD164 silencing on glioma cells is sustained in vivo, CD164-silenced and non-silenced U87 cells were 
A
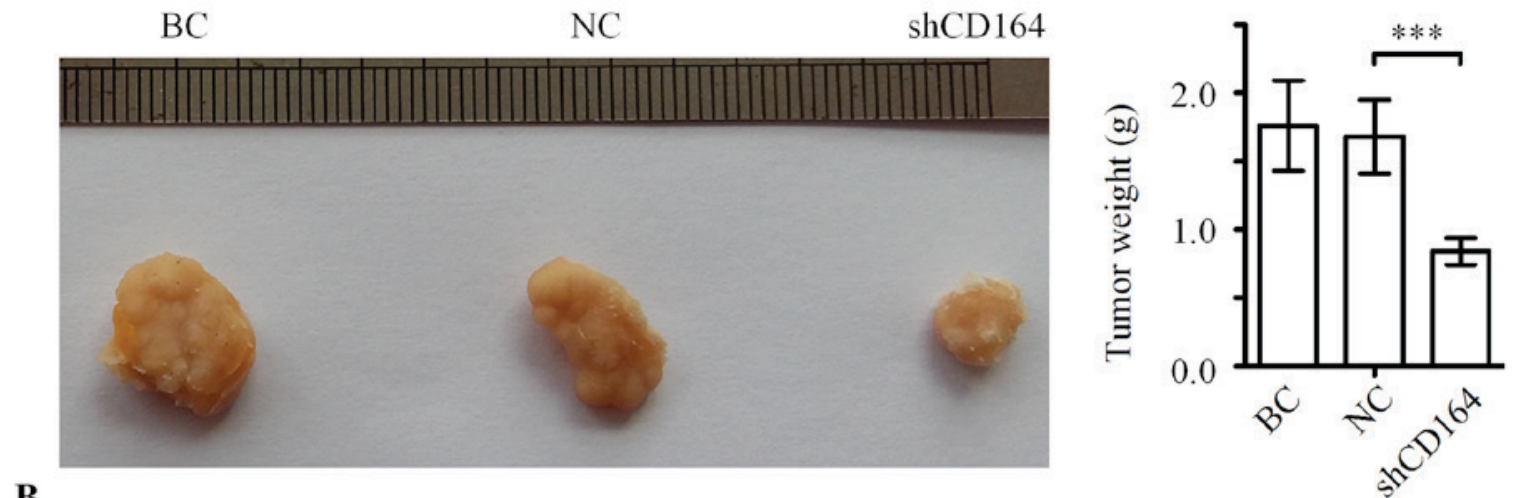

B

$\mathrm{BC}$

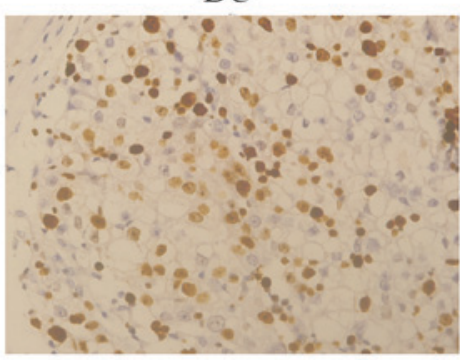

C

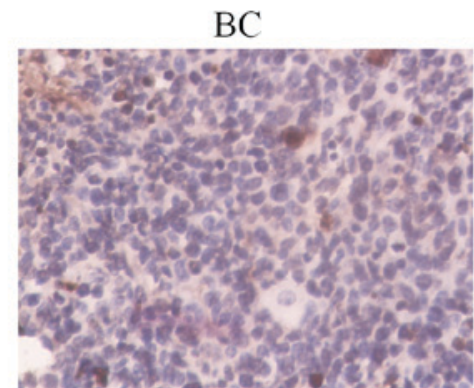

$\mathrm{NC}$

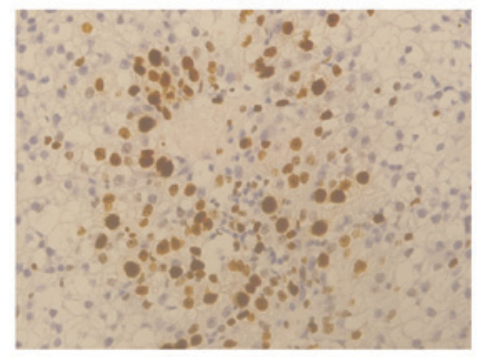

$\mathrm{NC}$

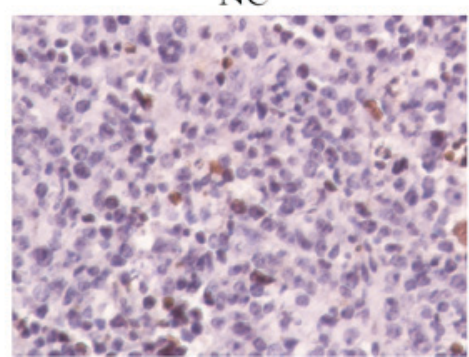

$\operatorname{shCD} 164$

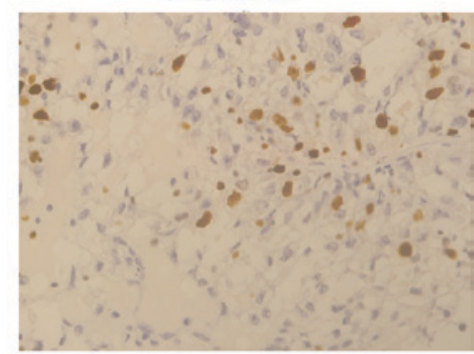

$\operatorname{shCD} 164$

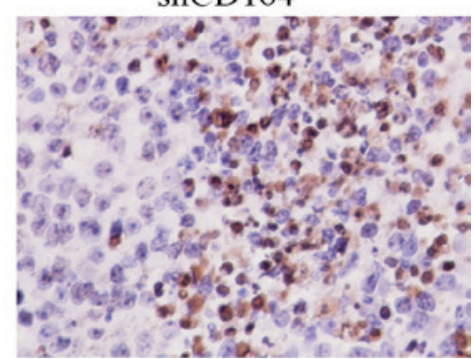

Figure 5. The antitumor effect of CD164 in vivo. (A) Necropsy photographs of subcutaneous tumors revealing the therapeutic benefit of CD164 knockdown. ${ }^{* * * *} \mathrm{P}<0.001$ vs. NC. (B) Representative images of immunohistochemistry of Ki-67 in the subcutaneous xenograft tissues (magnification, $\left.\mathrm{x} 400\right)$. (C) The number of TUNEL-positive nuclei in U87-shCD164 tumors was visibly increased when compared with the BC or NC groups (magnification, x400). CD164, cluster of differentiation 164; NC, negative control; BC, blank control; shCD164, CD164-specific short hairpin RNA.

A

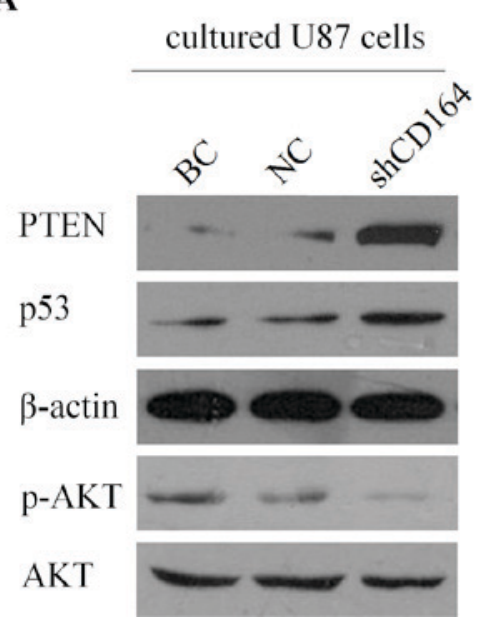

B

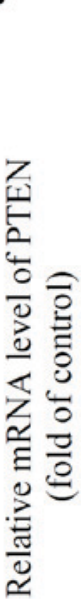

cultured U87 cells

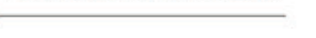

Figure 6. CD164 shRNA upregulated PTEN and inhibited the phosphoinositide 3-kinase/AKT signaling pathway in U87 cells. (A) Western blot analysis was performed to detect the levels of PTEN and its downstream target proteins following transfection with shCD164 in U87 cells. (B) PTEN mRNA expression levels were detected by reverse transcription-quantitative polymerase chain reaction in cultured U87 cells. (C) Western blot analysis demonstrating upregulated PTEN and p53 protein expression levels in U87-shCD164 xenograft tumor tissues. ${ }^{* * *} \mathrm{P}<0.001$ vs. NC. CD164, cluster of differentiation 164 ; shRNA, short hairpin RNA; PTEN, phosphatase and tensin homolog; p-AKT, phosphorylated protein kinase B; shCD164, CD164-specific short hairpin RNA; NC, negative control; BC, blank control. 
subcutaneously injected into mice. The in vivo results of the present study were similar to the in vitro results. CD164 knockdown significantly inhibited tumor growth in vivo. These results are consistent with the observed decrease in $\mathrm{Ki}-67$ immunoreactivity and increased TUNEL staining observed in the xenograft tumors. Therefore, the in vitro and in vivo results of the present study indicated that CD164 dysfunction may lead to decreased glioma tumor growth.

In order to investigate the molecular mechanisms underlying the CD164-associated promotion of tumorigenesis in glioma, the expression PTEN in glioma was detected. During normal tissue development, PTEN functions as an essential regulator of cell proliferation, apoptosis, migration and differentiation (17). Furthermore, PTEN is an established tumor suppressor gene that possesses dual-specificity phosphatase activities. Dysregulation of PTEN in mice results in the development of multiple solid tumors, and depletion of PTEN promotes the development of multiple cancers $(23,24)$. The PI3K/AKT signaling pathway is an intracellular signaling pathway, and activation of this pathway has been observed in a variety of tumors. Phosphorylation of AKT exerts anti-apoptotic effects by regulating downstream substrates, including Bax and Bcl2. Loss of PTEN results in hyperactivation of PI3K/AKT pathway (25). In addition, previous studies have revealed that PTEN is implicated in glioma; however, the regulation of PTEN during glioma progression remains unclear $(26,27)$. Consistent with these observations, the results of the present study have provided novel evidence demonstrating that the expression of PTEN at the mRNA and protein level was increased in response to CD164 depletion. The observed upregulation in PTEN expression was associated with decreased AKT phosphorylation, and an increase in p53 expression, as well as the growth inhibition of glioma cells in vitro. Notably, the in vitro results were confirmed in vivo. These results implied that the tumorigenic effects of CD164 in the progression of glioma may be dependent on the PTEN/PI3K/AKT signaling pathway.

In conclusion, the present study revealed for the first time, that the expression of CD164 may be involved in the tumorigenesis of glioma via the PTEN/PI3K/AKT signaling pathway. These results provide an improved understanding of the mechanisms of tumorigenesis in glioma, and CD164 may therefore present a novel candidate therapeutic target for the treatment of patients with glioma.

\section{References}

1. Rodon J, Carducci MA, Sepulveda-Sánchez JM, Azaro A, Calvo E, Seoane J, Braña I, Sicart E, Gueorguieva I, Cleverly AL, et al: First-in-human dose study of the novel transforming growth factor- $\beta$ receptor I kinase inhibitor LY2157299 monohydrate in patients with advanced cancer and glioma. Clin Cancer Res 21: 553-560, 2015.

2. Peters KB, West MJ, Hornsby WE, Waner E, Coan AD, McSherry F, Herndon JE II, Friedman HS, Desjardins A and Jones LW: Impact of health-related quality of life and fatigue on survival of recurrent high-grade glioma patients. J Neurooncol 120: 499-506, 2014.

3. Liu H, Lv Z and Guo E: Knockdown of long noncoding RNA SPRY4-IT1 suppresses glioma cell proliferation, metastasis and epithelial-mesenchymal transition. Int J Clin Exp Pathol 8: 9140-9146, 2015.

4. Chen XH, Ling XM and Shi S: microRNA-106a induces the proliferation and apoptosis of glioma cells through regulating JNK/MAPK pathway. Eur Rev Med Pharmacol Sci 19: 3412-3417, 2015.
5. Kwiatkowska A and Symons M: Signaling determinants of glioma cell invasion. Adv Exp Med Biol 986: 121-141, 2013.

6. Havens AM, Jung Y, Sun YX, Wang J, Shah RB, Bühring HJ, Pienta KJ and Taichman RS: The role of sialomucin CD164 (MGC-24v or endolyn) in prostate cancer metastasis. BMC Cancer 6: 195, 2006.

7. Darash-Yahana M, Pikarsky E, Abramovitch R, Zeira E, Pal B, Karplus R, Beider K, Avniel S, Kasem S, Galun E and Peled A: Role of high expression levels of CXCR4 in tumor growth, vascularization and metastasis. FASEB J 18: 1240-1242, 2004.

8. Tabaczar S, Domeradzka K, Czepas J, Piasecka-Zelga J, Stetkiewicz J, Gwoździński K and Koceva-Chyła A: Anti-tumor potential of nitroxyl derivative Pirolin in the DMBA-induced rat mammary carcinoma model: A comparison with quercetin. Pharmacol Rep 67: 527-534, 2015.

9. Murad N, Kokkinaki M, Gunawardena N, Gunawan MS, Hathout Y, Janczura KJ, Theos AC and Golestaneh N: miR-184 regulates ezrin, LAMP-1 expression, affects phagocytosis in human retinal pigment epithelium and is downregulated in age-related macular degeneration. FEBS J 281: 5251-5264, 2014.

10. Forde S, Tye BJ, Newey SE, Roubelakis M, Smythe J, McGuckin CP, Pettengell R and Watt SM: Endolyn (CD164) modulates the CXCL12-mediated migration of umbilical cord blood CD133+ cells. Blood 109: 1825-1833, 2007.

11. Doyonnas R, Yi-Hsin Chan J, Butler LH, Rappold I, Lee-Prudhoe JE, Zannettino AC, Simmons PJ, Bühring HJ, Levesque JP and Watt SM: CD164 monoclonal antibodies that block hemopoietic progenitor cell adhesion and proliferation interact with thefirst mucin domain of the CD164 receptor. J Immunol 165: 840-851, 2000.

12. Jorgensen-Tye B, Levesque JP, Royle L, Doyonnas R, Chan JY, Dwek RA, Rudd PM, Harvey DJ, Simmons PJ and Watt SM: Epitope recognition of antibodies that define the sialomucin, endolyn (CD164), a negative regulator of haematopoiesis. Tissue Antigens 65: 220-239, 2005.

13. Lin J, Xu K, Wei J, Heimberger AB, Roth JA and Ji L: MicroRNA-124 suppresses tumor cell proliferation and invasion by targeting CD164 signaling pathway in non-small cell lung cance. J Gene Ther 2: 6, 2016.

14. Shi JA, Lu DL, Huang X and Tan W: miR-219 inhibits the proliferation, migration and invasion of medulloblastoma cells by targeting CD164. Int J Mol Med 34: 237-243, 2014.

15. Tang J, Zhang L, She X, Zhou G, Yu F, Xiang J and Li G: Inhibiting CD164 expression in colon cancer cell line HCT116 leads to reduced cancer cell proliferation, mobility, and metastasis in vitro and in vivo. Cancer Invest 30: 380-389, 2012.

16. Havens AM, Jung Y, Sun YX, Wang J, Shah RB, Bühring HJ, Pienta KJ and Taichman RS: He role of sialomucin CD164 (MGC-24v or endolyn) in prostate cancer metastasis. BMC Cancer 6: 195, 2006.

17. Coustan-Smith E, Song G, Clark C, Key L, Liu P, Mehrpooya M, Stow P, Su X, Shurtleff S, Pui CH, et al: New markers for minimal residual disease detection in acute lymphoblastic leukemia. Blood 117: 6267-6676, 2011.

18. Chirumbolo S: CD164 and other recently discovered activation markers as promising tools for allergy diagnosis: What's new? Clin Exp Med 11: 255-257, 2011.

19. Livak KJ and Schmittgen TD: Analysis of relative gene expression data using real-time quantitative PCR and the 2(-Delta Delta C(T)) Method. Methods 25: 402-408, 2001.

20. Huang AF, Chen MW, Huang SM, Kao CL, Lai HC and Chan JY: CD164 regulates the tumorigenesis of ovarian surface epithelial cells through the SDF-1 $\alpha /$ CXCR4 axis. Mol Cancer 12: 115, 2013.

21. Kordezangeneh M,IraniS,Mirfakhraie R,Esfandyari-Manesh M, Atyabi F and Dinarvand R: Regulation of BAX/BCL2 gene expression in breast cancer cells by docetaxel-loaded human serum albumin nanoparticles. Med Oncol 32: 208, 2015.

22. Rodríguez-Berriguete $\mathrm{G}$, Torrealba $\mathrm{N}$, Ortega MA, Martínez-Onsurbe P, Olmedilla G, Paniagua R, Guil-Cid M, Fraile B and Royuela M: Prognostic value of inhibitors of apoptosis proteins (IAPs) and caspases in prostate cancer: Caspase-3 forms and XIAP predict biochemical progression after radical prostatectomy. BMC Cancer 15: 809, 2015.

23. Shang Y, Guo XX, Li WW, Rao W, Chen ML, Mu LN and Li SJ: Cucurbitacin-B inhibits neuroblastoma cell proliferation through up-regulation of PTEN. Eur Rev Med Pharmacol Sci 18: 3297-3303, 2014 
24. Li XT, Wang HZ, Wu ZW, Yang TQ, Zhao ZH, Chen GL, Xie XS, Li B, Wei YX, Huang YL, et al: miR-494-3p regulates cellular proliferation, invasion, migration and apoptosis by PTEN/AKT signaling in human glioblastoma cells. Cell Mol Neurobiol 35: 679-687, 2015

25. Ma Q, Zhang Y, Meng R, Xie KM, Xiong Y, Lin S, He ZL, Tao T, Yang Y, Zhao JZ and He JQ: MAGI3 suppresses glioma cell proliferation via upregulation of PTEN expression. Biomed Environ Sci 28: 502-509, 2015.
26. Wang LJ, He CC, Sui X, Cai MJ, Zhou CY, Ma JL, Wu L, Wang H, Han SX and Zhu Q: MiR-21 promotes intrahepatic cholangiocarcinoma proliferation and growth in vitro and in vivo by targeting PTPN14 and PTEN. Oncotarget 6: 5932-5946, 2015. 27. Huang WR, Chiu HC, Liao TL, Chuang KP, Shih WL and Liu HJ: Correction: avian reovirus protein p17 functions as a nucleoporin Tpr suppressor Leading to activation of p53, p21 and PTEN and inactivation of $\mathrm{PI} 3 \mathrm{~K} / \mathrm{AKT} / \mathrm{mTOR}$ and ERK signaling pathways. PLoS One 10: e0138627, 2015. 